\title{
Associação entre Bem-Estar Espiritual e Autoestima em Pessoas com Insuficiência Renal Crônica em Hemodiálise
}

\author{
Association between Spiritual Well-Being and Self-Esteem of People \\ with Chronic Kidney Failure in Hemodialysis
}

\author{
Erika de Cássia Lopes Chaves*, Thaynara Paola de Carvalho, Camila Csizmar Carvalho, \\ Cristiane da Silva Marciano Grasselli, Rogério Silva Lima, Fábio de Souza Terra \\ \& Denismar Alves Nogueira \\ Universidade Federal de Alfenas, Alfenas, MG, Brasil
}

\begin{abstract}
Resumo
Este estudo quantitativo teve por objetivo avaliar o bem-estar espiritual e a autoestima de pacientes com insuficiência renal crônica em tratamento hemodialítico e investigar a relação entre ambos. Foi desenvolvido em uma unidade de hemodiálise de um hospital filantrópico do Sul de Minas Gerais, com amostra por conveniência $(N=118)$, predominância de mulheres e média de idade de 57 anos. A coleta de dados deu-se por meio de entrevista, de outubro a novembro de 2013, com utilização do instrumento de caracterização sociodemográfica, clínica e espiritual, da Escala de Avaliação da Autoestima de Rosenberg e da Escala de Bem Estar Espiritual. Foi aplicada estatística descritiva, coeficiente de correlação de Spearman e o teste de Mann-Whitney. A média de autoestima foi de 31,70, classificada como alta e o bem-estar espiritual, 92,91, classificado como moderado. Houve correlação estatística significativa ao nível de $1 \%$, ou seja, quanto maior foi o nível de bem estar espiritual encontrado, maior foi a autoestima. Além disso, quanto maior foi a importância que o indivíduo denotou à religiosidade/espiritualidade maior foi seu nível de autoestima e de bem-estar espiritual $(p=0,001)$. Dessa forma, pode-se considerar a espiritualidade como um recurso possível a ser considerado no enfrentamento da condição crônica e seu tratamento.

Palavras-chave: Insuficiência renal crônica, doenças renais, espiritualidade, autoestima.

Abstract

This quantitative study evaluates the spiritual well-being and self-esteem of patients with chronic renal failure on hemodialysis and investigates the relationship between them. Developed in a hemodialysis unit of a philanthropic hospital in the South of Minas Gerais, with convenience sample $(N=118)$, predominance of women and mean age of 57 years. Data collection was carried out through interviews between October-November 2013, using the sociodemographic, clinical and spiritual instrument, the Rosenberg Self-Esteem Rating Scale and Welfare Spiritual Scale. Descriptive statistics, Spearman correlation coefficient and the Mann-Whitney test were applied. The average self-esteem was 31.70, classified as high, and the spiritual well-being, 92.91, rated as moderate. There was a statistically significant correlation at $1 \%$, that is, the greater the level of spiritual well-being found, the higher the self-esteem. In addition, the higher the importance religiosity / spirituality for the individual, the higher their level of self-esteem and spiritual well-being $(p=.001)$. Thus, the use of spirituality can be considered as a coping strategy to face the disease and treatment.

Keywords: Chronic renal failure, kidney disease, spirituality, self-esteem.
\end{abstract}

A espiritualidade é integrada por valores, princípios, crenças e força interior, tem um caráter universal, subjetivo, multidimensional, de transcendência, sendo experi-

\footnotetext{
* Endereço para correspondência: Universidade Federal de Alfenas, Escola de Enfermagem, Rua Rua Gabriel Monteiro da Silva, 714, Centro, Alfenas, MG, Brasil 37130-000. E-mail: echaves@unifal-mg.edu.br, thaynara_sja@hotmail.com, camilacsizmar@yahoo.com. br, csmgrasselli@gmail.com, rogerio.lima@unifal-mg. edu.br, fabio.terra@unifal-mg.edu.br e denismar@ unifal-mg.edu.br
}

mentada individualmente (Lepherd, 2014; Szaflarski et al., 2014). O bem estar espiritual é definido como uma sensação de bem-estar experimentada quando se encontra um propósito que justifique o comprometimento com algo da vida (Ellison, 1983).

Um bom nível de espiritualidade e um elevado bem- estar espiritual podem repercutir positivamente na vida da pessoa, contribuindo para o desenvolvimento de harmonia, de paz, de força interior e de realizações, como proposto pelo mapa conceitual de espiritualidade de Lepherd (2014). 
Nessa ótica, entende-se que o bem estar espiritual pode refletir nos níveis de satisfação pessoal, e a melhor relação para consigo pode favorecer a melhoria da autoestima e contribuir para o enfrentamento de circunstâncias adversas, como o processo de adoecimento crônico e as consequências de sua terapêutica (Mosquera \& Stobaus, 2006). Por sua vez a autoestima pode ser compreendida como o sentimento, o apreço e a consideração que uma pessoa tem em relação a ela mesma (Dini, Quaresma, \& Ferreira, 2004) e pode ser concebida como um fator de proteção contra os possíveis malefícios gerados pela condição de doença (Juth, Smyth, \& Santuzzi, 2008). Apesar do bem estar e da autoestima serem dimensões de importância reconhecida por estudiosos da área da saúde, a sua relação ainda não foi bem estabelecida, fato esse corroborado pela escassez de pesquisas na literatura científica. Desse modo, acredita-se que são necessários estudos que se proponham a investigar essa correlação e seus desdobramentos nas diversas circunstâncias da assistência à saúde, particularmente no enfrentamento das condições crônicas.

Entre as diversas situações de adoecimento, merece ênfase a doença renal crônica, posto que ocasione desgaste à pessoa, determine modificações no cotidiano, gere complicações resultantes do tratamento e favoreça o aparecimento de sintomas relacionados à ansiedade (Terra et al., 2010). A hemodiálise (HD), a despeito de constituir uma forma indispensável de tratamento que prolonga a vida (Terra et al., 2010), implica em impactos negativos que incidem desde mudanças de ordem física relacionadas, por exemplo, à fístula arteriovenosa, às repercussões de ordem psíquica, como as alterações na autoestima, na imagem corporal, e o desenvolvimento de depressão (Cabral et al., 2013, Murray, Dobbels, Lonsdale, \& Harden, 2014). Desse modo, em face aos condicionantes da doença renal crônica e do seu tratamento, a espiritualidade pode representar um recurso adicional de enfrentamento (Davison, \& Jhangri, 2010; Hodge, 2006).

Nesse aspecto, ressalta-se a necessidade de uma assistência integral à pessoa com insuficiência renal crônica (IRC) em HD, que coloque em perspectiva suas necessidades, inclusive quanto à sua dimensão espiritual, para que sejam favorecidas as condições de enfrentamento da enfermidade e o seu processo terapêutico (Bezerra, Ribeiro, Sousa, Costa, \& Batista, 2012).

Nessa direção, considera-se que são oportunos estudos que se proponham a elucidar a relação entre o bem-estar espiritual e a autoestima de pessoas em condições crônicas e em tratamentos que modificam seu estilo de vida, com vistas a subsidiar reflexões a respeito das possibilidades de articulação dos recursos apresentados pela pessoa, como a espiritualidade, no processo do cuidado, numa perspectiva que extrapole a dimensão biológica dos modelos assistenciais hegemônicos e considere a pessoa como ator responsável pelo próprio cuidado.

Diante do exposto, este estudo teve como objetivo avaliar o bem-estar espiritual e a autoestima de pacientes com IRC em HD e investigar a relação entre ambas.

\section{Método}

Estudo com abordagem metodológica quantitativa, analítica, de caráter descritivo e de corte transversal, desenvolvido em uma unidade de HD de um hospital geral filantrópico no sul de Minas Gerais, onde são atendidos aproximadamente 160 pacientes.

\section{Participantes}

O estudo contou com uma amostragem por conveniência, formada por 118 pacientes com IRC em tratamento de HD, que atenderam aos critérios de elegibilidade: idade igual ou superior a 18 anos; pacientes orientados em tempo, espaço e pessoa; que se expressavam verbalmente e que não possuíam problemas auditivos. Esses dois últimos critérios foram adotados uma vez que a coleta de dados deu-se por meio de entrevista, realizada durante as sessões de HD.

\section{Instrumentos}

A coleta de dados foi realizada nos meses de outubro e novembro de 2013, utilizando-se um instrumento para caracterização sociodemográfica, clínica e espiritual composto com informações acerca de sexo, idade, escolaridade, realização de atividade para se distrair, ou seja, lazer, vida social, atividade e prática religiosa, importância dada à religiosidade/espiritualidade e dados clínicos como tempo de descoberta da IRC e tempo de tratamento.

Também foi aplicada a Escala de Avaliação da Autoestima de Rosenberg (Rosenberg, 1985) traduzida e adaptada no Brasil por Dini et al. (2004) cujo alfa de Cronbach foi 0,85 . Essa escala possui 10 itens designados a avaliar o nível de autoestima, cinco itens expressam sentimentos positivos (valores invertidos na pontuação) e cinco expressam sentimentos negativos. Possui respostas tipo Likert, de quatro pontos, que variam de "concordo plenamente" a "discordo plenamente". Os valores na somatória dos itens podem variar de $10 \mathrm{a} 40$, com altos valores indicando elevada autoestima (Rosenberg, 1985).

Neste estudo a Escala de Avaliação da Autoestima de Rosenberg apresentou boa consistência interna e adequabilidade para mensurar o fenômeno investigado (alfa de Cronbach de 0,86).

Foi aplicada também a Escala de Bem-estar Espiritual (EBE) criada por Paloutizian e Ellison (1982), validada e adaptada para o Brasil por Marques, Sarriera e Dell'Aglio (2009). Trata-se de uma escala do tipo Likert que objetiva avaliar o bem estar espiritual. É constituída por 20 itens subdivididos em duas subescalas, uma de bem estar religioso e outra de bem estar existencial. Esses itens são respondidos por uma escala que varia de "concordo fortemente" a "discordo fortemente". Os itens referentes ao bem estar religioso contêm uma referência a Deus, e os de bem estar existencial não possuem tal referência. Como pontos de corte para o escore geral de bem estar espiritual são sugeridos os intervalos de 20 a 40, 41 a 99 e 100 a 120, para baixo, moderado e alto bem estar espiritual, 
Chaves, E. C. L., Carvalho, T. P., Carvalho, C. C., Grasselli, C. S. M., Lima, R. S., Terra, F. S. \& Nogueira, D. A. (2015). Associação entre Bem-Estar Espiritual e Autoestima em Pessoas com Insuficiência Renal Crônica em Hemodiálise.

respectivamente (Paloutizian \& Ellison, 1982; Volcan, Souza, Mari, \& Horta, 2003).

Esta escala mostrou-se com boa consistência interna e adequada para mensurar o fenômeno investigado uma vez que para o bem-estar espiritual encontrou-se um alfa de Cronbach de 0,92; para bem-estar existencial, de 0,86 e para bem-estar religioso, 0,89 .

\section{Procedimentos de Coleta de Dados}

A coleta de dados deu-se por meio de entrevista e foi realizada durante as sessões de HD. No hospital filantrópico geral há o setor de HD composto por três salas grandes em que os pacientes permanecem sentados em suas cadeiras durante o tratamento de HD, há sessões no período matutino e no vespertino. Durante a realização do tratamento os pacientes foram convidados a participar da pesquisa. Nesse ambiente foram aplicados os instrumentos de pesquisa por meio de entrevista.

\section{Análise de Dados}

Os dados coletados foram organizados em planilha eletrônica utilizando-se o programa Microsoft Office
Excel 2007 e analisados pelo programa Statistical Package for the Social Sciences (SPSS), versão 23,0. Para descrever e resumir os resultados alcançados foi utilizada a estatística descritiva. Para o estudo analítico foram utilizados o coeficiente de correlação de Spearman e o teste Mann-Whitney. Considerou-se para todos os testes $5 \%$ de nível de significância. Além destes, utilizou-se o alfa de Cronbach para avaliação da consistência interna dos dois instrumentos.

\section{Aspectos Éticos}

Em respeito aos aspectos éticos, a pesquisa foi avaliada e aprovada pelo Comitê de Ética em Pesquisa (CEP; CAAE $\left.\mathrm{n}^{\mathrm{o}} 17149113.0 .0000 .5142\right)$

\section{Resultados}

As principais características que descrevem o perfil sociodemográfico dos participantes deste estudo são apresentadas na Tabela 1. A média de idade dos pacientes foi de 57 anos, desvio padrão de $\pm 14,29$.

Tabela 1

Perfil Sociodemográfico dos Participantes do Estudo, Alfenas, Minas Gerais, 2013 (N=118)

\begin{tabular}{|c|c|c|c|}
\hline \multicolumn{2}{|c|}{ Características investigadas } & \multirow{2}{*}{$\frac{f}{52}$} & \multirow{2}{*}{$\frac{\%}{44,1}$} \\
\hline \multirow{2}{*}{ Sexo } & Masculino & & \\
\hline & Feminino & 66 & 55,9 \\
\hline \multirow{5}{*}{ Renda familiar mensal* } & 1 & 46 & 39,0 \\
\hline & 2 a 3 & 60 & 50,8 \\
\hline & Até 5 & 7 & 5,9 \\
\hline & Entre 5 e 10 & 3 & 2,5 \\
\hline & Mais que 10 & 2 & 1,7 \\
\hline \multirow{6}{*}{ Nível de escolaridade } & Nunca estudou & 15 & 12,7 \\
\hline & Fundamental incompleto & 76 & 64,4 \\
\hline & Ensino médio & 16 & 13,6 \\
\hline & Ensino superior incompleto & 3 & 2,7 \\
\hline & Ensino superior completo & 8 & 6,8 \\
\hline & Pós-graduação completa & 1 & 0,8 \\
\hline \multirow{3}{*}{ Ocupação } & Desenvolve atividade remunerada & 13 & 11 \\
\hline & Aposentado & 98 & 83 \\
\hline & Dependente da família & 7 & 5,9 \\
\hline
\end{tabular}

Nota. *Em salários mínimos (valor R \$724,00). 
Em relação ao tempo em que os entrevistados realizavam tratamento hemodialítico, observou-se uma média de quatro anos e quatro meses e desvio padrão $\pm 4,58$. Dos participantes, $66,1 \%$ faziam tratamento há menos de cinco anos, $19,5 \%$ de cinco a dez anos e $14,9 \%$ há mais de dez anos.
A investigação quanto à religiosidade/espiritualidade apontou maioria com religião católica, mais de $90 \%$ (Tabela 2).

Com a aplicação dos instrumentos foi revelada uma autoestima elevada e um moderado bem-estar espiritual (Tabela 3).

\section{Tabela 2}

Caracterização quanto à Religiosidade/Espiritualidade dos Participantes do Estudo, Alfenas, Minas Gerais, 2013 $(N=118)$

\begin{tabular}{cccc}
\hline & Características investigadas & $f$ & $\%$ \\
\hline \multirow{2}{*}{ Religião } & Não, mas espiritualizado & 3 & 2,5 \\
& Católico & 91 & 77,1 \\
& Espírita & 1 & 0,8 \\
& Evangélico & 23 & 19,5 \\
Praticante & Sim & 85 & 72,0 \\
& Não & 33 & 28,0 \\
& & & \\
Importância da religiosidade/ & Não é importante & 4 & 3,4 \\
espiritualidade & Pouco importante & 5 & 4,2 \\
& Importante & 33 & 28,0 \\
& Muito importante & 76 & 64,4 \\
\hline
\end{tabular}

Tabela 3

Dados Estatísticos do Escore de Autoestima e de Bem-Estar Espiritual, Alfenas, Minas Gerais, 2013 (N=118)

\begin{tabular}{ccccccc}
\hline Variável & $\begin{array}{c}\text { Intervalo de } \\
\text { valores possíveis }\end{array}$ & $\begin{array}{c}\text { Intervalo de valores } \\
\text { encontrados }\end{array}$ & Mediana & Média & $\begin{array}{c}\text { Desvio } \\
\text { padrão }\end{array}$ & $r^{*}$ \\
\hline Autoestima & $10-40$ & $17-40$ & 33,00 & 31,70 & 5,38 & - \\
Bem-estar espiritual & $20-120$ & $50-120$ & 93,00 & 92,91 & 16,95 & $0,667^{* *}$ \\
Bem-estar existencial & $10-60$ & $19-60$ & 43,00 & 42,98 & 9,67 & $0,684^{* *}$ \\
Bem-estar religioso & $10-60$ & $17-60$ & 51,00 & 49,92 & 8,62 & $0,554^{* *}$ \\
\hline
\end{tabular}

Notas. *Coeficiente de correlação de Spearman em relação a Escala de Autoestima. **Significativo ao nível de $1 \%$.

Além disso, observaram-se correlações estatísticas significativas entre autoestima, bem estar espiritual geral, existencial e religioso. Dessa forma, quanto maior foi o bem estar espiritual da pessoa, maior foi a autoestima encontrada (Tabela 3). Também foi investigada a associação entre o perfil de religiosidade da pessoa com a autoestima e o bem-estar espiritual, conforme Tabela 4.

Observou-se que a escolaridade apresentou correlação com autoestima, ou seja, quanto maior o nível de escolaridade maior foi a autoestima encontrada.
Constatou-se também que quanto maior foi a importância que o indivíduo denotou a religiosidade/espiritualidade maior foi seu nível de autoestima e de bem-estar espiritual (Tabela 5).

\section{Discussão}

A espiritualidade, nesse estudo, despontou-se como um aspecto importante na vida dos pacientes em HD praticantes de sua religiosidade. As duas dimensões são 
Chaves, E. C. L., Carvalho, T. P., Carvalho, C. C., Grasselli, C. S. M., Lima, R. S., Terra, F. S. \& Nogueira, D. A. (2015). Associação entre Bem-Estar Espiritual e Autoestima em Pessoas com Insuficiência Renal Crônica em Hemodiálise.

Tabela 4

Relação entre Prática Religiosa, Autoestima e Bem Estar Espiritual, Alfenas, Minas Gerais, 2013 (N=118)

\begin{tabular}{ccc}
\hline & \multicolumn{2}{c}{ Prática Religiosa } \\
\cline { 2 - 3 } Variável & Rank Médio & $p^{*}$ \\
Autoestima & Sim $-62,67$ & 0,10 \\
& Não $-51,33$ & $0,01^{* *}$ \\
Bem-estar espiritual & & Sim $-64,25$ \\
Bem-estar existencial & Não $-47,27$ & $0,03 * *$ \\
& Sim $-63,74$ & Não $-48,59$ \\
Bem-estar religioso & Sim $-63,91$ & $0,02^{* *}$
\end{tabular}

Notas. *Teste de Mann-Whitney a 5\%. **Significativo a 5\%.

Tabela 5

Correlação da Escolaridade e Importância dada a Religião com Autoestima e Bem Estar Espiritual, Alfenas, Minas Gerais, $2013(N=118)$

\begin{tabular}{ccccc}
\hline & \multicolumn{2}{c}{ Escolaridade } & \multicolumn{2}{c}{ Importância dada à religião } \\
\cline { 2 - 5 } Variável & $r^{*}$ & $p$ & $r^{*}$ & $p$ \\
\hline Autoestima & 0,319 & 0,001 & 0,423 & 0,001 \\
Bem-estar espiritual & 0,174 & 0,06 & 0,459 & 0,001 \\
Bem-estar existencial & 0,133 & 0,15 & 0,420 & 0,001 \\
Bem-estar religioso & 0,209 & 0,02 & 0,442 & 0,001
\end{tabular}

Nota. *Coeficiente de Correlação de Spearman.

aspectos que podem favorecer a vivência da harmonia e do conforto e proporcionar consolo e o desenvolvimento de valores como o amor, a crença e a fé (Lepherd, 2014).

Nessa direção, a espiritualidade é uma dimensão humana que, associada à religiosidade, pode ser um recurso a ser mobilizado para o enfrentamento de situações adversas da vida. Diante da natureza existencial das modificações geradas pela doença renal crônica, a dimensão espiritual é capaz de desempenhar um papel importante na adaptação do paciente à sua condição, levando-o a desenvolver outra visão de si mesmo, de seu futuro e do meio em que vive (Davison \& Jhangri, 2010).

Lucchetti, Almeida e Granero (2010), demonstraram que pacientes com IRC em tratamento dialítico que possuíam menor nível de espiritualidade necessitavam de mais intervenções terapêuticas. Ademais, os autores concluí- ram que os pacientes com maior nível de espiritualidade utilizam da fé e da religião para encontrar apoio e alívio em sua dor.

No presente estudo pôde-se observar que os pacientes apresentaram nível moderado de bem-estar espiritual, conforme a classificação adotada (Paloutizian \& Ellison, 1982; Volcan et al., 2003). Entretanto, as pessoas praticantes de sua religiosidade tiveram seu bem estar espiritual mais elevado e a associação entre ambos foi estatisticamente significativa, apontando para uma estreita relação entre espiritualidade e religiosidade, uma vez que a prática religiosa pode servir como um canal para a expressão da espiritualidade (Davison \& Jhangri, 2010).

Autores demonstraram uma importante relação entre maior espiritualidade/religiosidade com a melhor qualidade e satisfação com a vida (Lucchetti et al., 2010). Por con- 
seguinte, a satisfação com a vida abarca a satisfação para consigo mesmo, que por sua vez implica na autoestima, a qual, neste estudo, apresentou-se com níveis elevados, diferentemente do que Murray et al. (2014) observaram para pessoas com IRC. Contudo, a autoestima esteve intimamente ligada ao bem-estar espiritual, ou seja, quanto maior foi o bem-estar espiritual maior foi a autoestima.

Nesse contexto, entende-se que a espiritualidade é importante para a autoestima da pessoa com doença renal crônica e pode interferir nas variadas dimensões da vida, conforme observado no estudo de Rocha e Fleck (2011) cujo objetivo foi investigar a associação entre a presença de uma doença crônica e a importância dada à religiosidade/ espiritualidade/crenças pessoais.

Por fim, este estudo corrobora os achados obtidos por outros pesquisadores que propõem a espiritualidade como recurso possível a ser considerado para favorecer o enfrentamento da condição crônica e, nessa travessia, contribuir para melhoria da autoestima da pessoa (Valcanti, Chaves, Mesquita, Nogueira, \& Carvalho, 2012; Yodchai, Dunning, Savage, Hutchinson, \& Oumtanee, 2014).

\section{Conclusão}

Os pacientes com IRC em tratamento hemodialítico, investigados neste estudo, apresentaram uma elevada autoestima e um moderado bem-estar espiritual. Além disso, constatou-se que quanto maior o bem-estar espiritual da pessoa, maior foi a sua autoestima.

Nesse sentido, as relações entre a espiritualidade, a autoestima e o processo de adoecimento crônico se apresentam como desafios a serem considerados no processo assistencial, tendo em conta que este não deve se ater aos aspectos biológicos, mas se desenvolver em uma perspectiva que leve em conta demais dimensões da pessoa.

Entretanto, como limitações deste estudo destaca-se a escassez na literatura de pesquisas que investigassem a relação entre a autoestima e o bem estar espiritual, o que permitiria uma compreensão mais adequada do fenômeno, por meio da comparação dos resultados. Destaca-se também, o fato de que maioria dos pacientes relatou possuir alguma religião ou ser espiritualizada, o que já pressupõe algum espaço para a espiritualidade e seus desdobramentos na autoestima.

Apesar dessas limitações não permitirem a generalização dos resultados, essa pesquisa abre espaço para que novos estudos busquem investigar as relações entre a autoestima e do bem-estar espiritual.

\section{Referências}

Bezerra, M. L. R., Ribeiro, P. R. S., Sousa, A. A., Costa, A. I. S., \& Batista, T. S. (2012). Diagnósticos de enfermagem conforme a teoria do autocuidado de Orem para pacientes em tratamento hemodialítico. Revista Ciência em Extensão, 8(1), 60-81. Recuperado em http://ojs.unesp.br/index.php/ revista_proex/article/view/533
Cabral, L. C., Trindade, F. R., Branco, F. M. F. C., Baldoino, L. S., Silva, M. L. R., \& Lago, E. C. A. (2013). A percepção dos pacientes hemodialíticos frente à fístula arteriovenosa. Revista Interdisciplinar, 6(2), 15-25. Recuperado em http:// revistainterdisciplinar.uninovafapi.edu.br/index.php/revinter/ article/view/43

Davison, S. N., \& Jhangri, G. S. (2010). Existential and supportive care needs among patients with chronic kidney disease. Journal of Pain and Symptom Management, 40(6), 838-843. doi:10.1016/j.jpainsymman.2010.03.015

Dini, G. M., Quaresma, M. R., \& Ferreira, L. M. (2004). Adaptação cultural e validação da versão brasileira da Escala de Auto-estima de Rosenberg. Revista da Sociedade Brasileira de Cirurgia Plástica, 19(1), 47-52. Recuperado em www.rbcp. org.br/imageBank/PDF/19-01-04pt.pdf

Ellison, C. W. (1983). Spiritual well-being: Conceptualization and measurement. Journal of Psychology and Theology, 11(4), 330-340. Recuperado em http://journals.biola.edu/jpt/ volumes/11/issues/4/articles/330

Hodge, D. R. (2006). A template for spiritual assessment: A review of the JCAHO requirements and guidelines for implementation. Social Work, 51(4), 317-326. Recuperado em http://sw.oxfordjournals.org/content/51/4/317.full.pdf

Juth, V., Smyth, J. M., \& Santuzzi, A. M. (2008). How do you feel?: Self-esteem predicts affect, stress, social interaction, and symptom severity during daily life in patients with chronic illness. Journal of Health Psychology, 13(7), 884-894. doi:10.1177/1359105308095062

Lepherd, L. (2014). Spirituality: Everyone has it, but what is it? International Journal of Nursing Practice. doi:10.1111/ ijn. 12285

Lucchetti, G., Almeida, L. G. C., \& Granero, A. L. (2010). Espiritualidade no paciente em diálise: $\mathrm{O}$ nefrologista deve abordar? Jornal Brasileiro de Nefrologia, 32(1), 128-132. doi:10.1590/S0101-28002010000100020

Marques, L. F., Sarriera, J. C., \& Dell'Aglio, D. D. (2009). Adaptação e avaliação da Escala de Bem-estar Espiritual (EBE). Avaliação Psicológica, 8(2), 179-186.

Mosquera, J. J. M., \& Stobaus, C. D. (2006). Auto-imagem, auto-estima e auto-realização: Qualidade de vida na universidade. Psicologia, Saúde \& Doenças, 7(1), 83-88. Recuperado em www.scielo.oces.mctes.pt/pdf/psd/v7n1/v7n1a06.pdf

Murray, P. D., Dobbels, F., Lonsdale, D. C., \& Harden, P. N. (2014). Impact of end-stage kidney disease on academic achievement and employment in young adults: A mixed methods study. Journal of Adolescent Health, 55(4), 1-8. doi:10.1016/j.jadohealth.2014.03.017

Paloutizian, R., \& Ellison, C. (1982). Loneliness, spiritual wellbeing and the quality of life. In D. Peplau \& D. Perlman (Eds.), Loneliness: A sourcebook of current theory, research and therapy (pp. 224-235). New York: John Wiley and Sons.

Rocha, N. S., \& Fleck, M. P. A. (2011). Avaliação de qualidade de vida e importância dada a espiritualidade/religiosidade/ crenças pessoais (SRPB) em adultos com e sem problemas crônicos de saúde. Revista de Psiquiatria Clínica, 38(1), 1923. doi:10.1590/S0101-60832011000100005

Rosenberg, M. (1985). Self-concept and psychological wellbeing in adolescence. In R. Leach, The development of self (pp. 205-246). Orlando, FL: Academic Press.

Szaflarski, M., Kudel, I., Cotton, S., Leonard, A. C., Tsevat, J., \& Ritchey, P. N. (2014). Multidimensional assessment of spirituality/religion in patients with HIV: Conceptual framework and empirical refinement. Journal of Religion \& Health, 51(4), 1239-1260. doi:10.1007/s10943-010-9433-9 
Chaves, E. C. L., Carvalho, T. P., Carvalho, C. C., Grasselli, C. S. M., Lima, R. S., Terra, F. S. \& Nogueira, D. A. (2015). Associação entre Bem-Estar Espiritual e Autoestima em Pessoas com Insuficiência Renal Crônica em Hemodiálise.

Terra, F. S., Costa, A. M. D. D., Ribeiro, C. C. S., Nogueira, C. S., Prado, J. P., Costa, M. D., ...de Morais, A. M. (2010). O portador de insuficiência renal crônica e suas dependências ao tratamento hemodialítico: Compreensão fenomenológica. Revista Brasileira de Clínica Médica, 8(4), 306-310. Recuperado em http://files.bvs.br/upload/S/1679-1010/2010/ v8n4/a003.pdf

Valcanti, C. C., Chaves, E. C. L., Mesquita, A. C., Nogueira, D. A., \& Carvalho, E. C. (2012). Coping religioso/espiritual em pessoas com doença renal crônica em tratamento hemodialítico. Revista da Escola de Enfermagem da USP, 46(4), 838-845. doi:10.1590/S0080-62342012000400008

Volcan, S. M. A., Souza, P. L. R., Mari, J. J., \& Horta, B. L. (2003). Relação entre bem-estar espiritual e transtornos psiquiátricos menores: Estudo transversal. Revista de Saúde Pública, 37(4), 440-445. doi:10.1590/S0034-89102003000400008

Yodchai, K., Dunning, T., Savage, S., Hutchinson, A. M., \& Oumtanee, A. (2014). How do Thai patients receiving haemodialysis cope with pain? Journal of Renal Care, 40(3), 205-215. doi:10.1111/jorc.12073 